\title{
Experimental Study on the Compatibility and Characteristics of a Dual-Target Microbubble Loaded with Anti-miR-33
}

\author{
Chen Yuan ${ }^{1, *}$ \\ Yanhong Li ${ }^{1, *}$ \\ Liyun Liu' \\ Baihetiya Tayier' \\ Lingjie Yang ${ }^{\prime}$ \\ Lina Guan ${ }^{1-3}$ \\ Yuming $\mathrm{Mu}$ (iD $^{1-3}$ \\ 'Department of Echocardiography, First \\ Affiliated Hospital of Xinjiang Medical \\ University, Urumqi, 8300I I, People's \\ Republic of China; ${ }^{2}$ State Key Laboratory \\ of Pathogenesis, Prevention and \\ Treatment of High Incidence Diseases in \\ Central Asia, First Affiliated Hospital of \\ Xinjiang Medical University, Urumqi, \\ People's Republic of China; ${ }^{3}$ Xinjiang Key \\ Laboratory of Medical Animal Model \\ Research, Clinical Medical Research \\ Institute of First Affiliated Hospital of \\ Xinjiang Medical University, Urumqi, \\ People's Republic of China \\ *These authors contributed equally to \\ this work
}

Objective: To prepare a new type of dual-target microbubble loaded with anti-miR-33 (ANM33).

Methods: Carrier core nanobubbles (NBs) were prepared by thin film hydration, and microbubbles loaded with PM1 (PCNBs) were prepared by grafting DSPE-PEG2000maleimide-PM1 onto the NB surface. ANM33 was connected via electrostatic adsorption and covalent bonding, and hyaluronic acid (HA) was covalently connected. PM1 and HA were the targets, and ANM33 was the intervention drug. To evaluate the general physical and chemical properties of the prepared dual-target microbubbles loaded with ANM33 (HAPANBs), we observed their morphology, particle size and surface potential while monitoring their stability and in vitro imaging ability, evaluated their toxic effect on cells and verified their ability to target cells.

Results: HA-PANBs had a regular morphology and good stability. The average particle size measured by a Malvern potentiometer was $1421.75 \pm 163.23 \mathrm{~nm}$, and the average surface potential was $-5.51 \pm 1.87 \mathrm{mV}$. PM1 and ANM33 were effectively connected to the NBs. The PM1, ANM33, and HA binding reached $89.0 \pm 1.1 \%, 65.02 \pm 5.0 \%$, and $61.4 \pm 3.5 \%$, respectively, and the maximum binding reached $2 \mu \mathrm{g}, 5 \mu \mathrm{g}$, and $7 \mu \mathrm{g} / 10^{8}$ microbubbles, respectively. HA-PANBs had no obvious toxic effects on cells, and their ability to continuously enhance imaging in vitro persisted for more than 15 minutes, obviously targeting foam cells in the early stage of AS.

Conclusion: HA-PANBs are ideal ultrasound contrast agents. The successful, firm connection of PM1 and HA to the NBs significantly increased the amount of carried ANM33. When microbubbles prepared with 2:4:7 PM1:ANM33:HA were used as a contrast agent, they had a high ANM33 carrying capacity, stable physical properties, and significantly enhanced imaging and targeting of foam cells in the early stage of AS.

Keywords: ultrasound microbubbles, molecular targeting, dual targeting, atherosclerosis

\section{Introduction}

The development of atherosclerosis (AS) involves a series of cellular and molecular events. ${ }^{1}$ Early macrophages can delay the progression of the disease through efferocytosis and cholesterol efflux. ${ }^{2,3}$ Recent studies have shown that miR-33 in macrophages inhibits the above functions. Through this inhibition, antisense oligonucleotides of miR-33 (ANM33) can reduce the level of miR-33 and effectively reduce the inflammatory response in plaques. ${ }^{4,5}$ However, using ANM33 to nonselectively inhibit miR-33 in systemic macrophages can cause serious side effects. ${ }^{6}$

\footnotetext{
Correspondence: Yuming Mu; Lina Guan Department of Echocardiography, The First Affiliated Hospital of Xinjiang Medical University, I37 Liyushan Road, Ürümqi, Xinjiang Uyghur, Autonomous Region, 830054, People's Republic of China

Email mym1234@|26.com;

sanjin_lsx@163.com
} 
With the development of molecular biology and ultrasound microbubble contrast agents, ultrasound molecular imaging is an emerging target for the prevention and treatment of cardiovascular events by detecting molecular markers and carrying therapeutic genes for targeted development and treatment of cardiovascular diseases. ${ }^{7}$ In previous studies, atherosclerosis (AS)-targeted ultrasound contrast agent with vascular cell adhesion molecule-1 (VCAM-1) monoclonal antibody prepared by the direct connection method has been proven to be successfully targeted to AS plaques, which is of certain value for noninvasive ultrasound evaluation of early $\mathrm{AS} .{ }^{8}$ However, most of the targeted agents are nonspecifically absorbed by the liver, and this off-target effect seriously affects the target seeking efficiency. The latest research shows that the targeting efficiency of contrast agents can be effectively improved by the combined application of different targets. ${ }^{9}$ Moreover, the function of macrophages plays an important role in the progression of AS disease. If the role of macrophages in inhibiting inflammation can be fully performed, the development of the disease can be alleviated.

Based on this, it is the key to further improve precise targeted delivery to identify AS through double-target multistage targeting and further locate the functionally impaired macrophages within the plaque. To achieve this goal, this experiment intends to use a variety of linking methods to develop and prepare a double-targeted lipid microbubble with ANM33 and aptamer PM1 on the surface of lipid microbubbles that are wrapped with a layer of HA. To explore the best compatibility between the two targets and the best load of antisense oligonucleotides, a firm and stable connection was obtained through a variety of connection methods. Microbubbles can penetrate blood vessel walls, remain stable in the circulatory system, and treat AS. The precise targeting of damaged macrophages and intracellular drug release provide a new and reliable method for the prevention and treatment of AS.

Therefore, this experiment intends to use a variety of connection methods to develop and prepare a new type of dual-target microbubble loaded with ANM33. The surfaces of nanobubbles (NBs) were modified with the aptamers PM1 and ANM33 and wrapped in a layer of HA.

\section{Materials and Methods}

\section{Materials and Instruments}

DSPC (Avanti Corporation, USA), DSPE-PEG2000 (Avanti Corporation, USA), DSPE-PEG2000-maleimide (Avanti Company, USA), DC-cholesterol·HCl (Avanti Company,
USA), DPPE (Avanti Company, USA), glycerol (Sigma, USA), sodium hyaluronate (HA, 200-400 kDa, Xi'an Ruixi), aptamer PM1 base sequence SH-CCCC TGCAGGTGATTTTGCTCAAGTCGTTCCCGTCCTCTCCTCTGCGCCCCGTCTCGGCCAGTATCGCTAATCAGGCGGAT-HS, (Shanghai Shenggong, China), a sequence with FITC-labelled or unlabelled at the $5^{\prime}$ end, ANM33 and ANM33 double-modified with cy3 and amino groups (Guangzhou Ruibo, China), DiI fluorescent dye (Yeasen, USA), RAW264.7-T1 macrophages (Procell Life Science \& Technology, China), oxidized low-density lipoprotein (oxLDL; Yiyuan Biotechnology, China), Oil red O staining kit (Sigma, China), high-glucose DMEM (ScienCell, China), and double antibodies (ScienCell, USA) were used. A fluorescence microscope (LEICA CTR6000, Germany), laser confocal microscope (Leica SP8, Germany), flow cytometer (Beckman, USA), and laser nanoparticle size and zeta potential analyser (Malvern, UK) were also used.

\section{Experimental Methods}

Preparation of Lipid Microbubbles

DSPC and DSPE-PEG2000 at a molar ratio of 9:1 were dissolved in chloroform to prepare a mother liquor; the test tube containing the liquor was placed above the vortex and flushed with nitrogen. During the process, a white film formed on the test tube wall. After nitrogen flushing, tris solution was added to dissolve the lipid film on the tube wall. After 5 minutes in a $65^{\circ} \mathrm{C}$ water bath, the hydration liquid was dispensed into vials. After evacuation for 45 minutes, perfluoropropane was poured into the vial. Finally, the vial was fixed in a silver mercury blender and shaken for 30 seconds to obtain a microbubble solution, which was placed in a refrigerator at $4^{\circ} \mathrm{C}$ for later use.

\section{Preparation and Loading of Dual-Target Microbubbles with ANM33 \\ Preparation of CNBs}

DSPC, DSPE-PEG2000, DSPE-PEG2000-maleimide, DPPC, DPPE, and DC-CHOL at a molar ratio of 9:0.5:0.5:9:2:1 were dissolved in chloroform (Table 1), and the remainder of the preparation was the same as that for ordinary lipid microbubbles.

Construction of Microbubbles Loaded with PMI (PCNBs) and Determination of the PMI Concentration

Preinsertion. Before inspection, PM1 and DSPE-PEG2000maleimide were mixed at a molar ratio of $1: 5$ and reacted 
Table I Percent Molar Ratio of the Lipid-Based Components of Several NBs

\begin{tabular}{|l|c|c|c|c|c|c|c|}
\hline Group & Surface Potential & DSPC & DPPC & DSPE & DC-chol & DPPE & DSPE-mal \\
\hline NBs & Negative charge & 9 & - & 0.5 & - & - & - \\
CNBs & Positive charge & 9 & - & 0.5 & 1 & - & - \\
PCNBs & Positive charge & 9 & - & 0.5 & 1 & - & 0.5 \\
PANBs & Positive charge & 9 & 9 & 0.5 & 1 & - & 0.5 \\
HA-PANBs & Negative charge & 9 & 9 & 0.5 & 1 & 2 & 0.5 \\
\hline
\end{tabular}

under $\mathrm{N}_{2}$ protection at $4^{\circ} \mathrm{C}$ for 24 hours to obtain PM1PEG2000-DSPE for processing.

Postinsertion. Before detection, the sulfhydryl group of PM1 was activated by referring to the method of Cordray et al. ${ }^{10}$ Then, $5 \mu \mathrm{L}, 10 \mu \mathrm{L}, 20 \mu \mathrm{L}, 30 \mu \mathrm{L}$, or $40 \mu \mathrm{L}$ of a solution containing $10 \mu \mathrm{M}$ sulfhydryl groups and AF488 double-modified PM1 and $5 \mu \mathrm{L}$ of $1 \mathrm{M}$ tris(2-carboxyethyl)phosphine (TCEP) were mixed and allowed to react at room temperature for 30 minutes. The PM1 solution was mixed with CNBs in a blender. PM1 and CNBs were incubated for 30 minutes and centrifuged at $400 \mathrm{~g}$ for 3 minutes, the subnatant was discarded, and the upper layer of microbubbles was washed with PBS solution three times to remove unbound PM1 and obtain PCNBs before placing in a refrigerator at $4^{\circ} \mathrm{C}$ for later use.

The PCNBs were diluted to $400 \mu \mathrm{L}$ with PBS and placed in a dedicated flow cytometer vial. Then, flow cytometry was performed to detect the rate of binding of PM1 to NBs in the PCNB complexes and to analyse the amount of PM1 bound in the PCNBs. Images were generated via laser confocal microscopy.

\section{Loading the Therapeutic Factor ANM33}

Construction of an ANM33 Standard Curve. ANM33 labelled with $\mathrm{Cy} 3$ was purchased as a pink powder and dissolved in RNase-free distilled water to prepare solutions at $0.00625,0.0125,0.025,0.05,0.1,0.2$, and 0.4 $\mathrm{nmol} / \mathrm{mL}$. An ultraviolet spectrophotometer was used to measure the UV absorption of the Cy3-labelled ANM33 solutions at an excitation wavelength of $\lambda=550 \mathrm{~nm}$ to construct a standard curve.

Determination of the Amount of ANM33 in PANBs. First, $5 \mu \mathrm{L}, 10 \mu \mathrm{L}, 20 \mu \mathrm{L}, 30 \mu \mathrm{L}$, and $40 \mu \mathrm{L}$ of $20 \mu \mathrm{M}$ Cy3labelled ANM33 were added to PCNBs and incubated for $30 \mathrm{~min}$ with shaking at room temperature. After centrifugation at $400 \mathrm{~g}$ for 3 minutes to pellet any remaining unbound ANM33, PCNBs carrying ANM33 on the surface (PANBs) were successfully prepared. After the reaction, the UV absorbance of the supernatant was measured. DL ANM33 $(\%)=\left[\left(\mathrm{OD}_{\mathrm{A}}-\mathrm{OD}_{0}\right) /\right.$ total number of microbubbles $]$ $\times 100 \%$, and $\mathrm{BE}_{\mathrm{ANM} 33}(\%)=\left[\left(\mathrm{OD}_{\mathrm{A}}-\mathrm{OD}_{0}\right) / \mathrm{OD}_{\mathrm{A}}\right] \times 100 \%$, where $\mathrm{OD}_{\mathrm{A}}$ and $\mathrm{OD}_{\mathrm{O}}$ are the total amounts of added and unbound ANM33, respectively. In this way, the amount of ANM33 carried by the NBs and the efficiency of ANM33 transport by the NBs were analysed to optimize the preparation conditions.

\section{Preparation of HA-Coated PCNBs}

Construction of HA-PANBs. The carboxyl groups of HA were activated by ultrasonically dispersing $20 \mathrm{mg}$ of HA into $10 \mathrm{~mL}$ of MES buffer. Then, $80 \mathrm{mg}$ of EDC and $120 \mathrm{mg}$ of preactivated NHS, which had both been stored at $4^{\circ} \mathrm{C}$ and then equilibrated to room temperature before use, were added, and the solution was sonicated for 15 s. The container was sealed with parafilm and shaken at $37^{\circ} \mathrm{C}$ for $15 \mathrm{~min}$ on a shaker. Then, $1 \mu \mathrm{g}, 3 \mu \mathrm{g}, 5 \mu \mathrm{g}, 7 \mu \mathrm{g}$, $9 \mu \mathrm{g}$, or $11 \mu \mathrm{g}$ of the activated HA was added to the PANBs to construct HA-coated PANBs (HA-PANBs).

Determination of the Amount of $\mathrm{HA}$ in HA-PANBs. A modified CTAB method was used. The appropriate concentration of HA solution was mixed with acetate buffer for a final volume of $1 \mathrm{~mL}$. Then, $2 \mathrm{~mL}$ of a $25 \mathrm{~g} / \mathrm{L}$ CTAB solution was added and mixed uniformly; the turbidity of the mixed solution was measured at $400 \mathrm{~nm}$ within 10 min. Through the relationship between turbidity and the HA concentration, the $\mathrm{HA}$ content in the solution was determined: $\mathrm{BE}_{\mathrm{HA}}(\%)=\left[\left(\mathrm{C}_{0}-\mathrm{C}\right) / \mathrm{C}_{0}\right] \mathrm{X} 100 \%$, and $\mathrm{DL}$ HA $(\%)=\left[\left(\mathrm{C}_{0}-\mathrm{C}\right) /\right.$ total number of $\left.\mathrm{NBs}\right] \times 100 \%$, where $\mathrm{C}_{0}$ and $\mathrm{C}$ are the amount of free $\mathrm{HA}$ and the initial amount of HA added.

\section{Cell Culture and Cell Model Construction Cell Culture}

RAW264.7 cells (MФs) were inoculated in high-glucose DMEM containing 10\% FBS and 1\% double antibodies, and the cells were cultured in an incubator at $37^{\circ} \mathrm{C}$, 
suitable humidity and $5 \% \mathrm{CO}_{2}$. Fresh medium was used every day, and the cells were observed under a light microscope. When the cell density reached $80 \sim 90 \%$, they could be passed or used for further experiments.

\section{Induce Foam Cell Formation}

The damaged cell model was constructed with reference to the study of Zhang et al. ${ }^{11}$ MФs were seeded into the well plate and cultured for $24 \mathrm{~h}$, and then the medium was replaced with M199 medium containing OX-LDL for 12 $\mathrm{h}$. The inoculation density of $\mathrm{M} \varphi$ was $2.0 \times 10^{4}$ cells $/ \mathrm{mL}$, and a blank control group was set. Cells in each group were used for subsequent experimental detection after the end of the experiment.

\section{Identification of Foam Cells}

Oil red O staining was used to display the generated foam cells. Oil red O staining resulted in red lipid droplets, and the cells with aggregated lipid droplets were identified as foam cells. The degree of foam cell formation was indicated by observing the amount of oil red. The red area of the lipid droplets was quantitatively analysed and counted through the colour distinction function in the Image processing software ImageJ, and then quantitative information was obtained.

\section{Detection of Microbubbles}

Before testing, $10 \mu \mathrm{L}$ of microbubbles was added to PBS to a final volume of $1 \mathrm{~mL}$ and mixed gently by pipetting.

\section{Physical and Chemical Properties}

(1) The shape and size of the microbubbles to be tested were observed through optical microscopy, fluorescence microscopy and transmission electron microscopy.

(2) An automatic dilution particle counter (AccuSizer 780) combined with a nanoparticle size potential analyser (Malvern Zetasizer Nano) was used to analyse the particle size, distribution, concentration, and surface zeta potential of the microbubbles to be tested.

(3) Microbubble stability test: Microbubbles were kept at room temperature $\left(25^{\circ} \mathrm{C}\right)$, and changes in the particle size, dispersion index and concentration were detected at 5 min, $15 \mathrm{~min}, 30 \mathrm{~min}, 45 \mathrm{~min}$ and $60 \mathrm{~min}$.

\section{Evaluation of the Effect of Contrast-Enhanced Ultrasound} According to a previous method developed by the research team, agarose mimics were prepared, and the contrast effect of the microbubbles was evaluated in vitro as follows. (1) Preparation of a $2 \%$ agar mimic: a. A suitable amount of agarose was added to an Erlenmeyer flask and mixed with deionized water to form a $2 \%$ agarose solution. $\mathrm{b}$. The solution was placed in a microwave oven to accelerate agarose dissolution until the solution was completely clear. c. The clear solution was poured into a $100 \mathrm{~mm}^{2}$ Petri dish, and a $200 \mu \mathrm{L}$ EP tube was inserted into the solution and fixed, taking care not to penetrate through to the bottom of the solution. d. After the solution solidified into a gel, the EP tube was slowly removed. At this point, the preparation of the lipid agar mimic with sample holes was completed, and the mimic was cut into strips with a blade for later use. (2) In vitro ultrasound imaging: Two hundred microlitres of NB solutions of different concentrations in each group were added to the agar mimics. A Vevo $2100^{\circledR}$ small-animal ultrasound imager with a probe frequency of $18 \mathrm{MHz}$ was used. After positioning in twodimensional mode, the imager was switched to contrast mode, ultrasound images were recorded and stored, and Qvevo image analysis software was used to evaluate the ultrasonic image quantitatively.

\section{Biosafety Assessment}

A CCK-8 kit was used to evaluate the cytotoxicity of the HA-PCNBs. RAW264.7 cells were cultured by adding 100 $\mu \mathrm{L}$ of cell suspension to a 96-well plate and preculturing the plate in an incubator for 24 hours $\left(37^{\circ} \mathrm{C}, 5 \% \mathrm{CO} 2\right)$. Then, $10 \mu \mathrm{L}$ of HA-PANBs at concentrations of $10^{8}, 10^{7}$, $10^{6}, 10^{5}$, or $10^{4}$ was added to the culture plate, which was incubated for $24 \mathrm{~h}, 48 \mathrm{~h}$, or $72 \mathrm{~h}$. Ten microlitres of CCK solution was added to each well, and the culture plate was incubated in the incubator for $1-4$ hours. The absorbance was measured at $450 \mathrm{~nm}$ with a microplate reader.

Cell survival rate $=[($ experimental well-blank well $) /($ control well-blank well)] $\times 100 \%$; experimental well (medium containing cells, CCK-8, and the test substance); control well (culture containing cells, CCK-8, but no test substance) blank hole (medium without cells, test substance, or CCK8). Cell survival rate $=[($ experimental well-blank well $) /($ control well-blank well) $\times 100 \%$; experimental well (medium containing cells, CCK-8, and the test substance); control well (culture containing cells, CCK-8, but no test substance) blank hole (medium without cells, test substance, or CCK-8).

\section{In vitro Targeting Capability Verification}

With the help of damaged MФs, the intracellular distribution and concentration of ANM33 were tracked in vitro to evaluate the effect of HA-PANBs on targeting damaged МФs.

After coincubation of fluorescence-labelled HAPANBs, confocal laser scanning microscopy and flow 
cytometry were used to detect fluorescence uptake in macrophages.

\section{Statistical Processing}

When the measurement data conformed to a normal distribution, they were represented as the means \pm standard deviations, and when they did not conform to a normal distribution, they were represented as medians and interquartile ranges. Statistical analysis mainly involved oneway analysis of variance (ANOVA) and independent sample $t$-tests. When the data did not conform to a normal distribution, a nonparametric test was used; repeated-measurement data analysis involved repeated-measurement analysis of variance. The confidence interval for determining differences between groups was set to $95 \%$, and differences were considered statistically significant when $\mathrm{P}<0.05$.

\section{Results}

\section{General Characteristics}

The microbubble solutions appeared as uniform curd-like suspensions; the microbubbles were circular, smooth, uniform in size, and evenly distributed under light and electron microscopy, as shown in Figure 1. The particle size of the NBs was measured by a laser nanoparticle size and zeta potential analyser to be approximately $(655.20 \pm 91.51) \mathrm{nm}$, with an average surface potential of $(-23.07 \pm 2.18) \mathrm{mV}$, a PDI of $(0.33 \pm 0.93)$, and a concentration of $(4.13 \pm 0.22)$ $\mathrm{x} 10^{8}$. The particle size of the HA-PANBs was approximately $(1421.75 \pm 163.23) \mathrm{nm}$, with an average surface potential of $(-5.51 \pm 1.87) \mathrm{mV}$, a PDI of $(0.33 \pm 0.56)$, and a concentration of $(2.72 \pm 0.71) \times 10^{8}$, as shown in Table 2 and Figure 2. Coating with HA was complete within $60 \mathrm{~min}$. The particle size and contrast intensity of the PANBs did not significantly change when coated with HA, as shown in Table 3.

Under the visual field of a fluorescent inverted microscope, AF488-labelled PM1 and DIL-labelled NBs in PCNBs appeared green and yellow, respectively, with different excitation wavelengths, while the NBs appeared red, indicative of DIL labelling. When the complexes were observed under a laser confocal scanning microscope, the AF488-labelled PM1 ultrasound microbubbles (ie, PANBs) were bright green, the Cy3-labelled ANM33 was bright red, and the fused product of the two emitted yellow fluorescence (Figure 3).

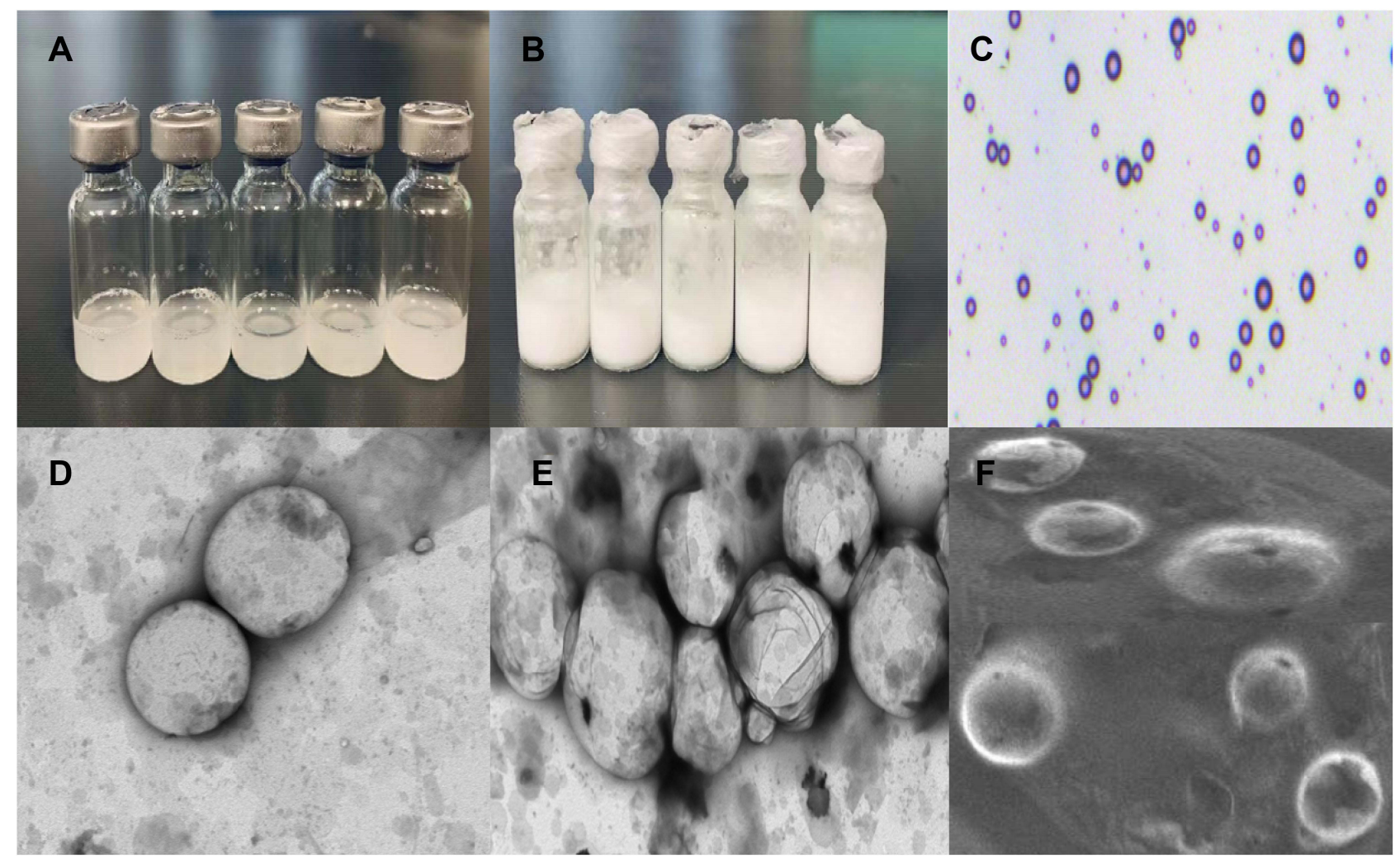

Figure I The morphology and structure of the NBs.

Notes: (A) The phospholipid suspension before mechanical vibration. (B) The phospholipid suspension after mechanical vibration. (C) Photomicrograph of NBs. (D) TEM image of NBS. (E) TEM image of HA-PANBs. (F) SEM image of HA-PANBs. 
Table 2 Particle Size, Dispersion Index and Concentration of NBs $(\mathrm{n}=5, \bar{\chi} \pm s)$

\begin{tabular}{|l|c|c|c|c|}
\hline Group & Particle Size (nm) & Polydispersity Index (PDI) & Potential & Concentration $\left(\times 1 \mathbf{0}^{\mathbf{8}}\right)$ \\
\hline NBs & $655.20 \pm 91.5 I$ & $0.33 \pm 0.93$ & $-23.07 \pm 2.18$ & $4.13 \pm 0.22$ \\
CNBs & $726.05 \pm 63.73$ & $0.33 \pm 0.13$ & $28.35 \pm 9.35$ & $3.52 \pm 0.60$ \\
PCNBs & $730.55 \pm 64.93$ & $0.32 \pm 0.08$ & $10.61 \pm 1.17$ & $3.77 \pm 0.50$ \\
PANBs & $795.10 \pm 66.64$ & $0.32 \pm 0.06$ & $6.92 \pm 0.58$ & $2.77 \pm 0.82$ \\
HA-PANBs & $1421.75 \pm 163.23$ & $0.33 \pm 0.56$ & $-5.5 I \pm 1.87$ & $2.72 \pm 0.71$ \\
\hline
\end{tabular}

The efficiency of connecting PM1 with the preinsertion method was $36.4 \pm 1.7 \%$, the efficiency of connecting PM1 with the postinsertion method was 80.0 $\pm 2.1 \%$, and the maximum binding capacity reached 2 $\mu \mathrm{g} / 10^{8}$ microbubbles, as shown in Figures 4 and 5 . The ANM33 amino group was modified by covalent connection and static electricity. The maximum adsorption rate was $62.7 \pm 2.1 \%$, and the maximum binding capacity was $5 \mu \mathrm{g} / 10^{8}$ microbubbles. The binding rate of ANM33 through only electrostatic adsorption was 44.1 $\pm 1.7 \%$, as shown in Figure 6 . The maximum binding rate of HA was $61.4 \pm 3.5 \%$, and the maximum binding capacity was $7 \mu \mathrm{g} / 10^{8}$ microbubbles, as shown in Figure 7.
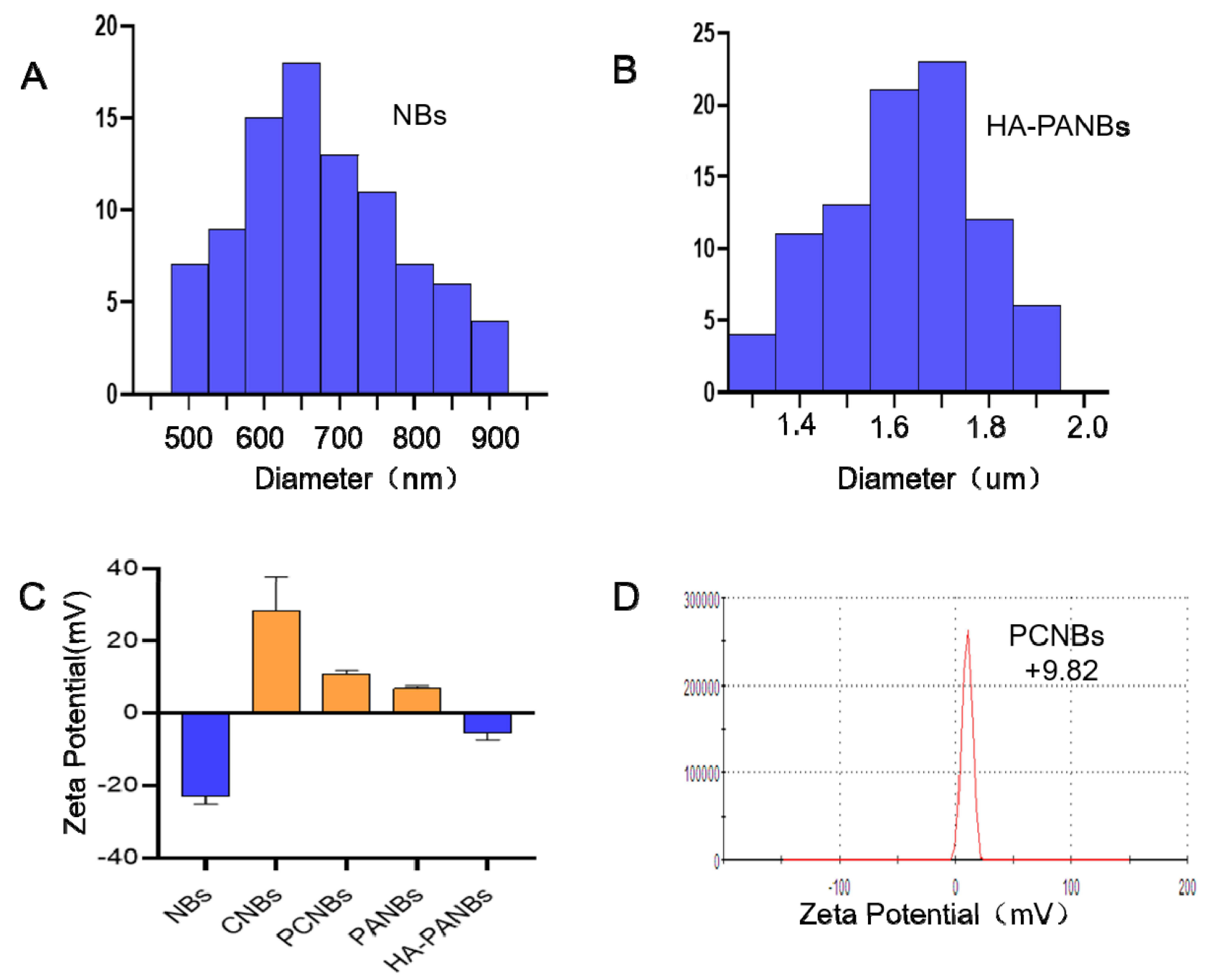

Figure 2 General characteristics of NBs.

Notes: (A) Size distribution of NBs. (B) Size distribution of HA-PANBs. (C) Zeta potential of several NBs. (D) Representative PCNBs Zeta Potential. 
Table 3 Changes in Particle Size and the Contrast Signal from HA-PANBs Over 60 Min

\begin{tabular}{|l|c|c|c|c|c|c|}
\hline index & $0 \mathrm{~min}$ & $\mathbf{5 m i n}$ & $15 \mathrm{~min}$ & $\mathbf{3 0 m i n}$ & $\mathbf{4 5 m i n}$ & $\mathbf{6 0 m i n}$ \\
\hline $\begin{array}{l}\text { Particle size } \\
(\mathrm{nm})\end{array}$ & $1614 \pm 93.18$ & $1632 \pm 75.07$ & $1684 \pm 89.24$ & $1681 \pm 101.08$ & $1667 \pm 101.18$ & $1678 \pm 123.69$ \\
\hline Contrast signal intensity (a.u.) & $34.66 \pm 1.40$ & $34.12 \pm 1.98$ & $34.85 \pm 2.40$ & $34.57 \pm 2.98$ & $34.21 \pm 2.32$ & $34.69 \pm 2.95$ \\
\hline
\end{tabular}

\section{Cell Viability}

When HA-PANBs were added to the 96-well plate at a concentration of $10^{4}$, the cell viability was $99.57 \pm$ $1.21 \%$. There was no significant difference in HAPANBs compared with the control group $109.4 \pm 2.11 \%$, and the viability remained at a relatively high level. When the concentration of HA-PANBs was gradually increased, the cell viability was $99.48 \pm 1.32 \%, 98.96 \pm 1.78 \%, 98.82$ $\pm 2.17 \%$, and $98.74 \pm 3.77 \%$. Moreover, HA-PANBs did not cause significant damage to cells $(\mathrm{P}>0.05)$. The above results demonstrate the safety of HA-PANBs in vitro, as shown in Figure 8.

\section{Damaged Mps Were Constructed in vitro} Identification of Foam Cells

To establish damaged MФs, Oil red O staining was observed to show intracellular lipid droplet formation even after $60 \mathrm{mg} / \mathrm{L}$ ox-LDL intervention, as shown in Figure 9A.

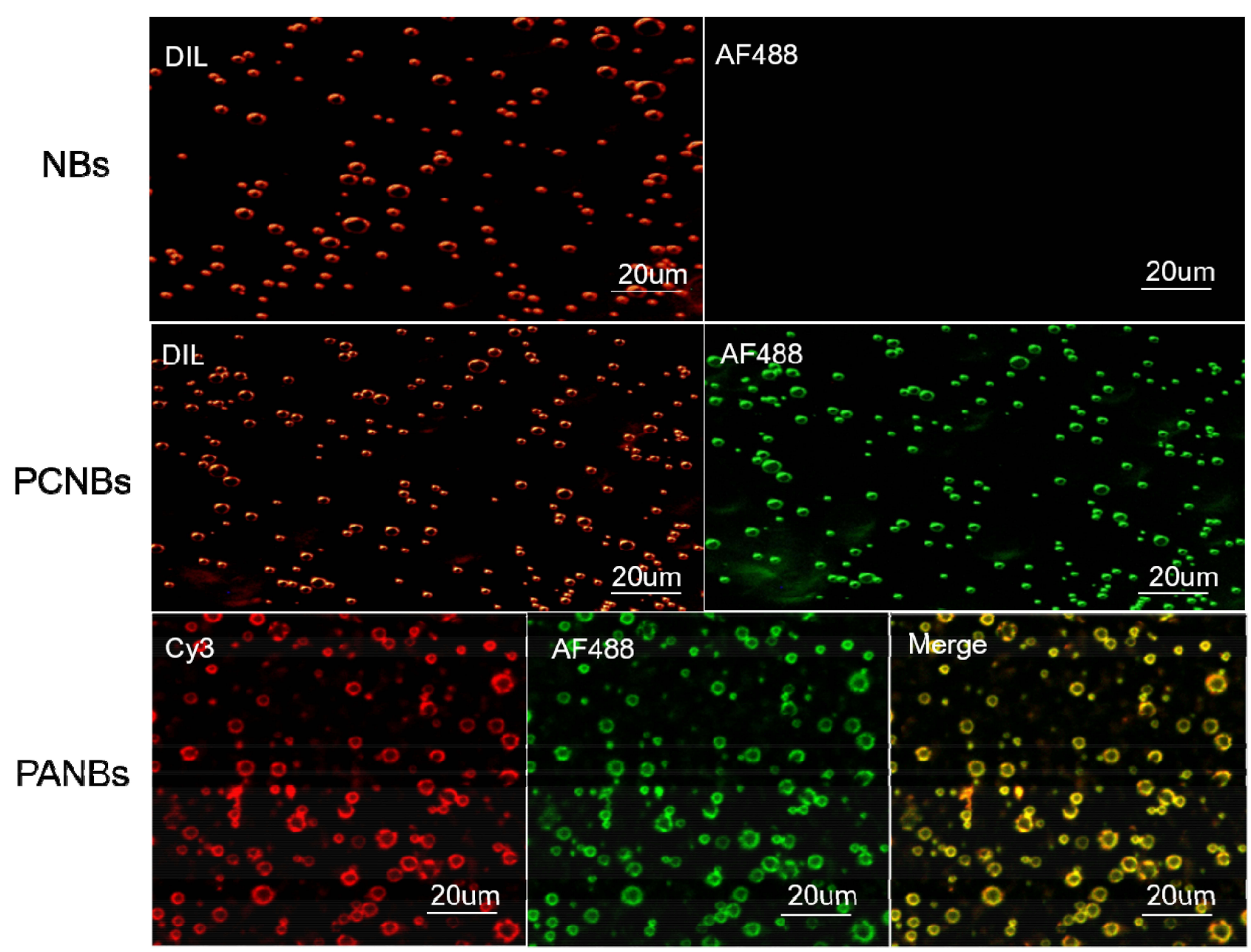

Figure 3 The combination of NBs and the aptamers PMI and ANM33.

Notes: Representative fluorescence micrographs of NBs and PCNBs, Red (Dil-labeled NBs); green (AF488-labeled PMI); Representative laser scanning confocal micrographs of PANBs. Red (Cy3-labeled ANM33); green (AF488-labeled PMI); yellow indicates the successful conjugation of PMI and anti-miR-33 to the surface of NBs. 

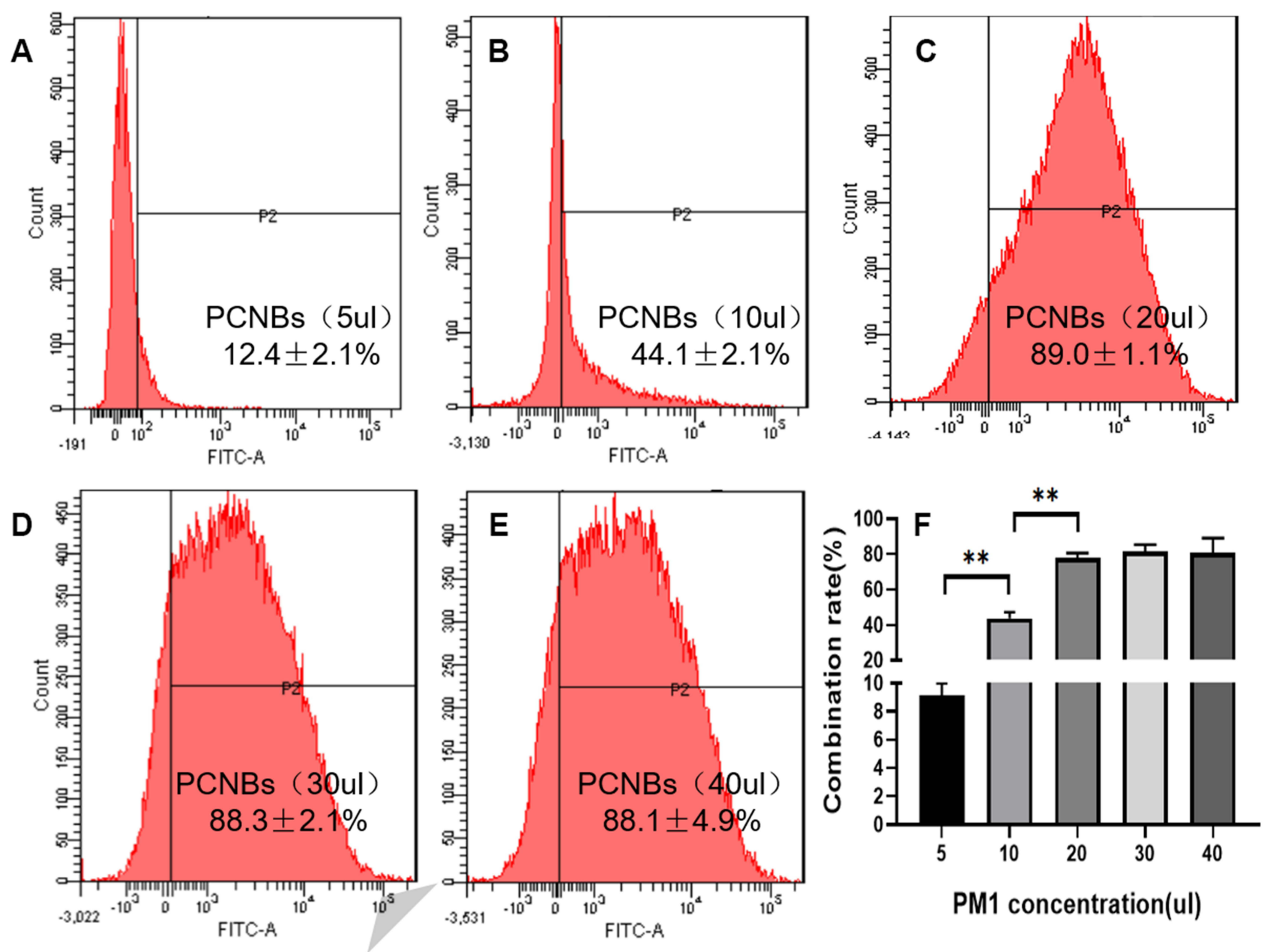

Figure 4 PMI conjugation efficiency based on flow cytometry. (A-E) The binding efficiency of PMI increased as the amount of PMI increased; F:quantitative results of conjugation efficiency. ${ }^{*} \mathrm{P}<0.00 \mathrm{I}$.

\section{Targeting Ability in vitro}

According to the experimental results of damaged $М \Phi s$ in the in vitro culture, a foam cell model was used for the in vitro targeting study. After coincubation with NBs and HA-PANBs, unbound free microbubbles were removed after 3 washes. As shown in Figure 9B, the number of microbubbles around each foam cell in the HA-PANBS group $14.5 \pm 2.46 \%$ was significantly higher than that in the NBs group \%2.30 $\pm 0.46(\mathrm{P}<0.05)$.

As shown in Figure 10, red fluorescence was expressed by CY3-labelled ANM33, and green fluorescence was expressed by AF488-labelled PM1 in each group of microbubbles. Confocal microscopy revealed a large number of red and green fluorescence signals around the HA-PANB-targeted foam cell group. Flow cytometry showed that the cell binding rate was 25.2 $\pm 1.7 \%$ in the HA-PANB group and $0.5 \pm 0.9 \%$ in the NB group after 30 minutes. After 24 hours, the transfection rate was $78.1 \pm 2.1 \%$ in the HA-PANB group and 9.4 $\pm 1.1 \%$ in the NB group.

\section{Evaluation of the Ultrasound Contrast \\ Enhancement in vitro}

When NBs and HA-PANBs were injected at different concentrations into phantom holes in agarose gel, the contrast intensity began to increase after 6 seconds and then remained constant for 15 minutes, as shown in Figure 11. The peak enhancement (au) for each concentration of HA-PANBs was $22.65 \pm 1.59,34.38 \pm 1.20,35.29 \pm 0.98,34.89 \pm 0.93$, and 40.36 \pm 1.35 , and there was no significant difference between the $10^{6}$ and $10^{7}$ concentrations $(\mathrm{P}>0.05)$ (Tables 4 and 5). This result suggests that the addition of the target did not have a significant impact on the contrast effect, as shown in Figure 10. 


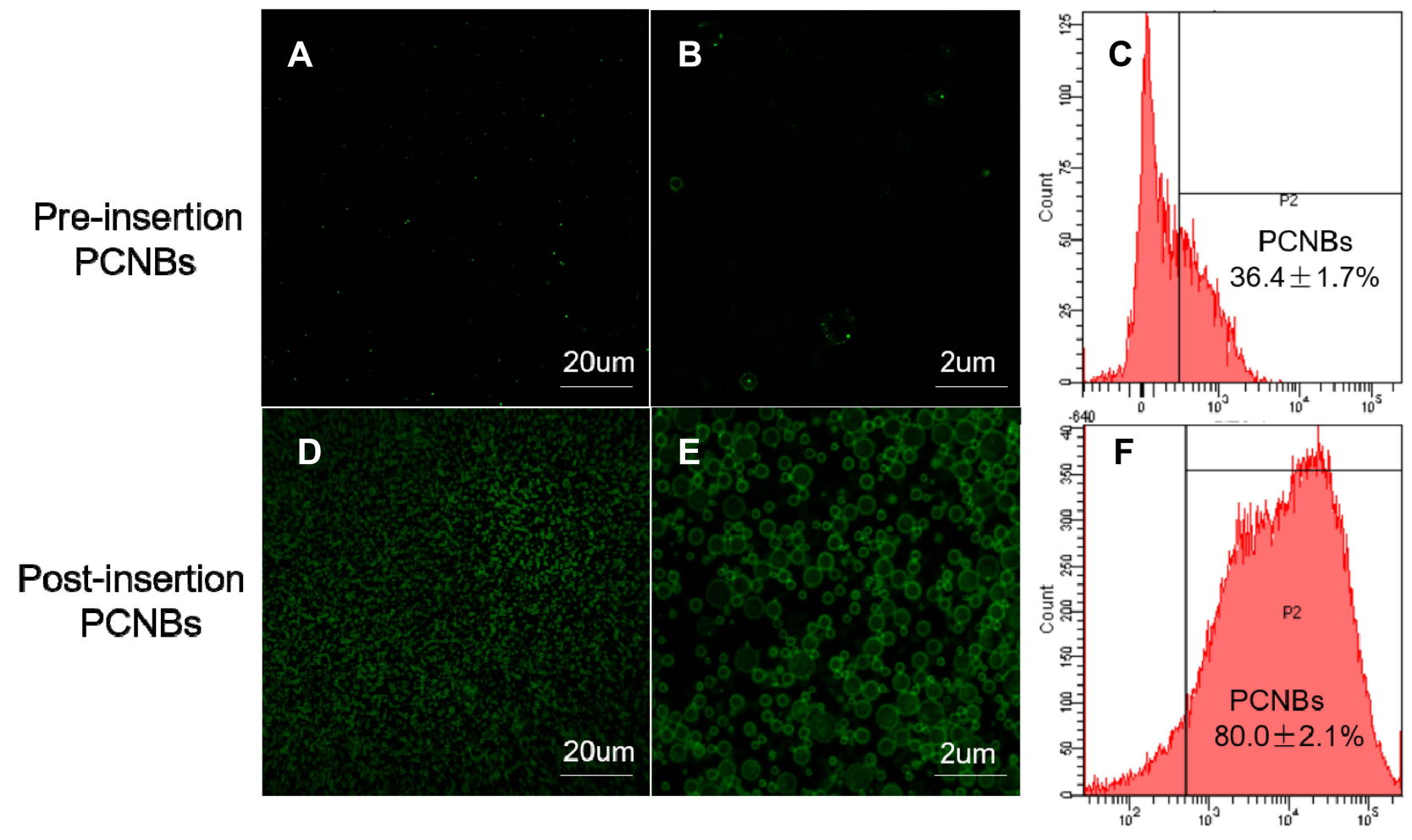

Figure 5 PMI conjugation efficiency influenced by the method of insertion. (A, B) Pre-insertion results of confocal laser microscopy at different magnifications; (D, E) Postinsertion results of confocal laser microscopy at different magnifications; (C,F) flow cytometry results of Pre-insertion and Post-insertion.
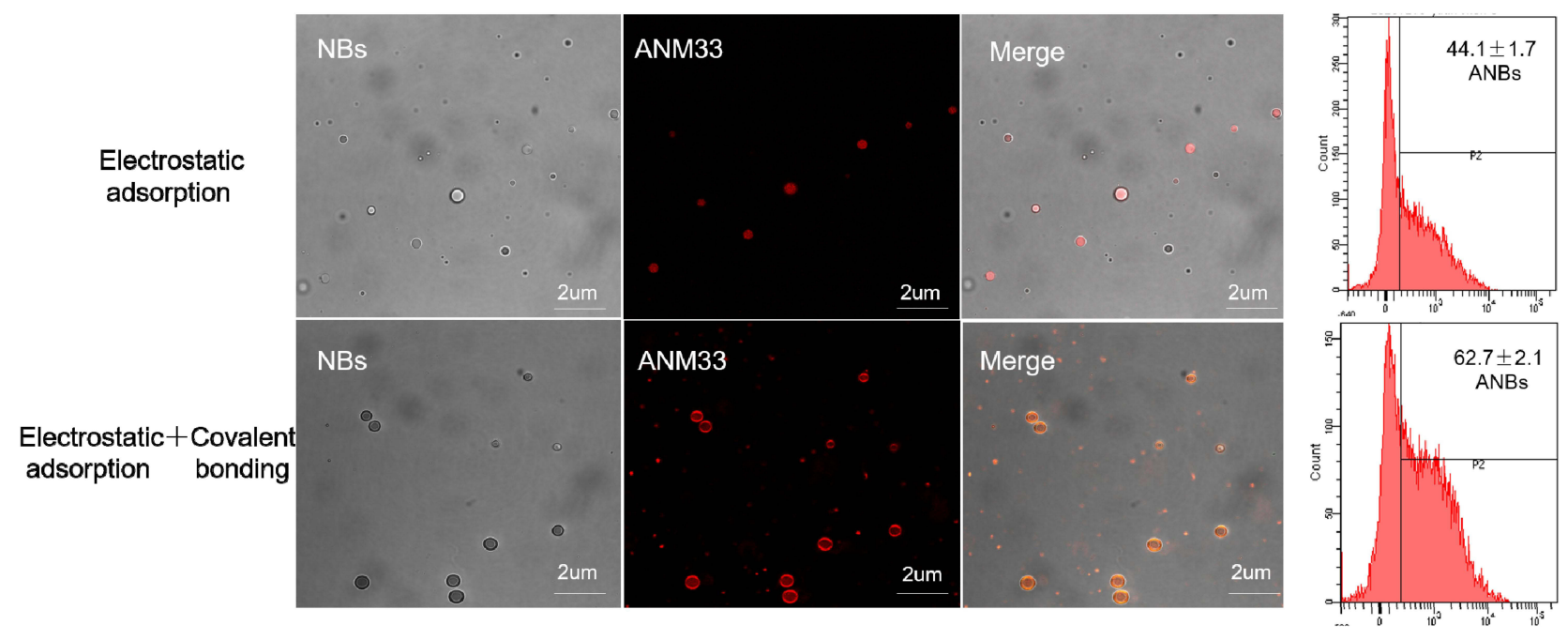

Figure 6 ANM33 conjugation efficiency influenced by the connection method.

\section{Discussion}

The formation of AS involves a series of cellular and molecular events. The latest research shows that the combined application of different targets can effectively improve the targeting efficiency of contrast agents, ${ }^{9,12,13}$ and macrophages play a role in the progression of AS disease.
Importantly, ${ }^{14,15}$ if macrophages can completely inhibit inflammation, they can arrest the development of the disease. At present, the methods of connecting ligands or antibodies to contrast agents mainly include physical adsorption, ionic bonding, biotin-avidin bridging, amino modification and sulfhydryl modification. ${ }^{16-18}$ Each connection method has 

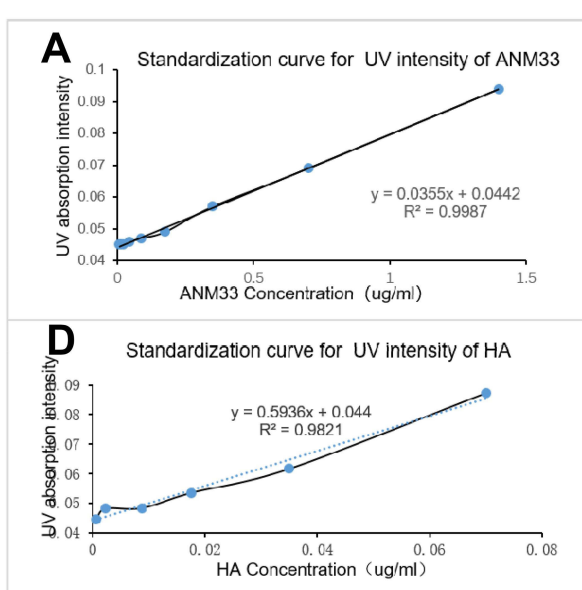
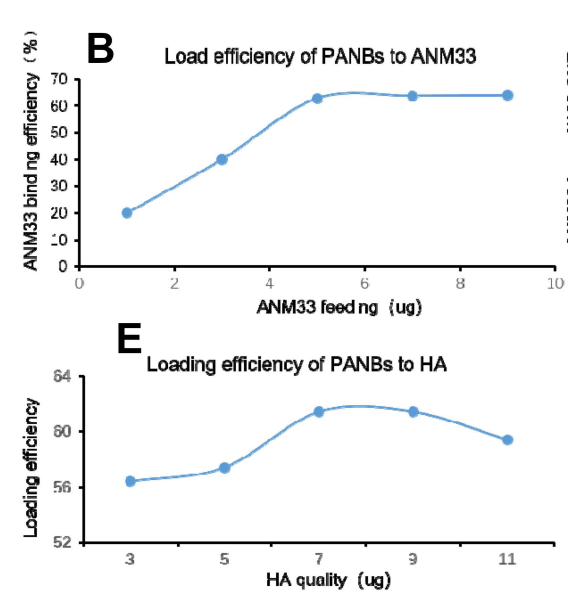
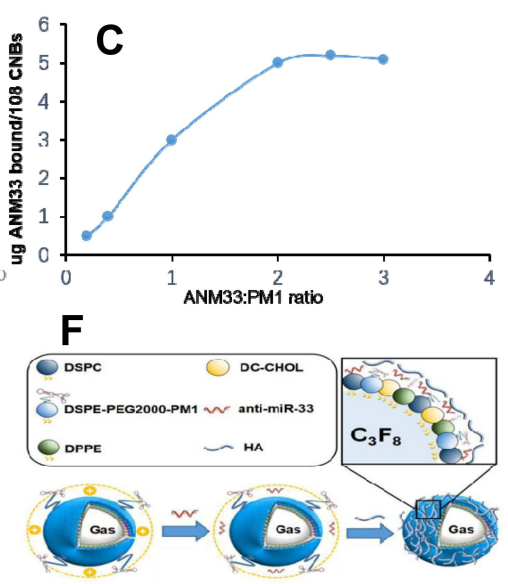

Figure 7 (A) UV absorption standard curve of Cy3-labeled ANM33. (B) Loading efficiency of ANM33 for different qualities. (C) The quality of loaded ANM33 after processing CNBs with different proportions of PMI. (D) UV absorption standard curve of HA; (E) loading efficiency of HA for different qualities. (F) Preparation of HAPANBs.

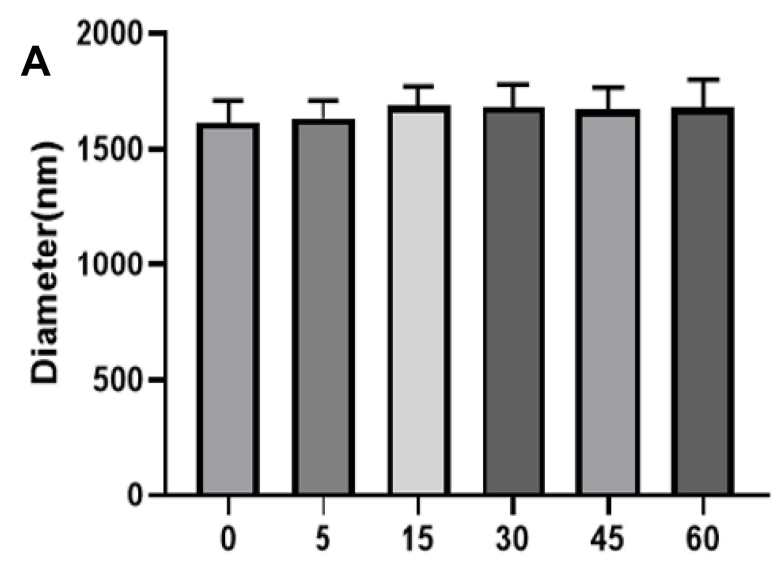

C

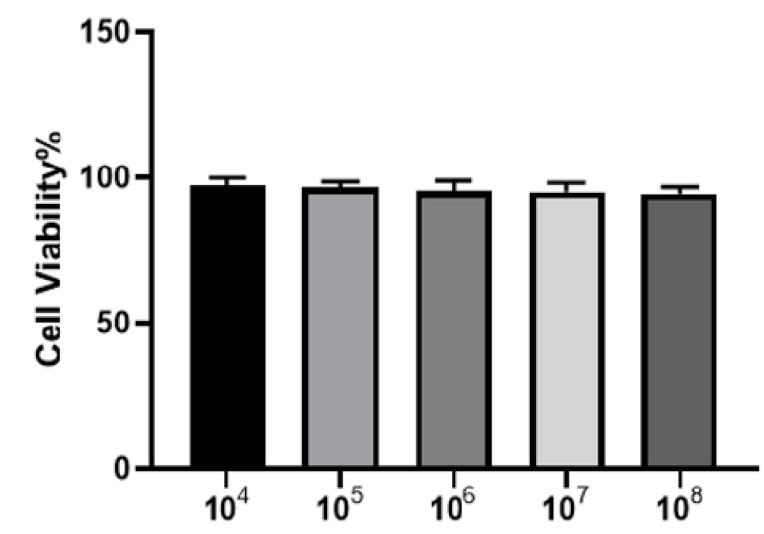

Concentration of HA-PANBs $(/ \mathrm{ml})$

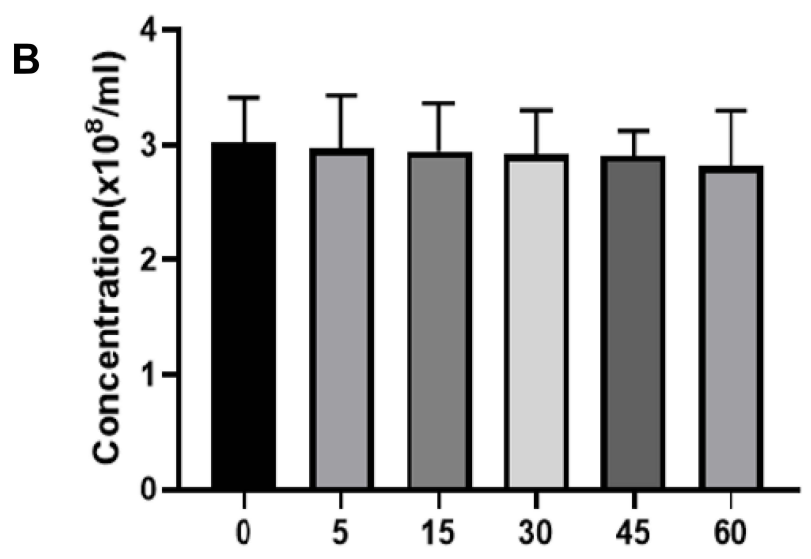

D
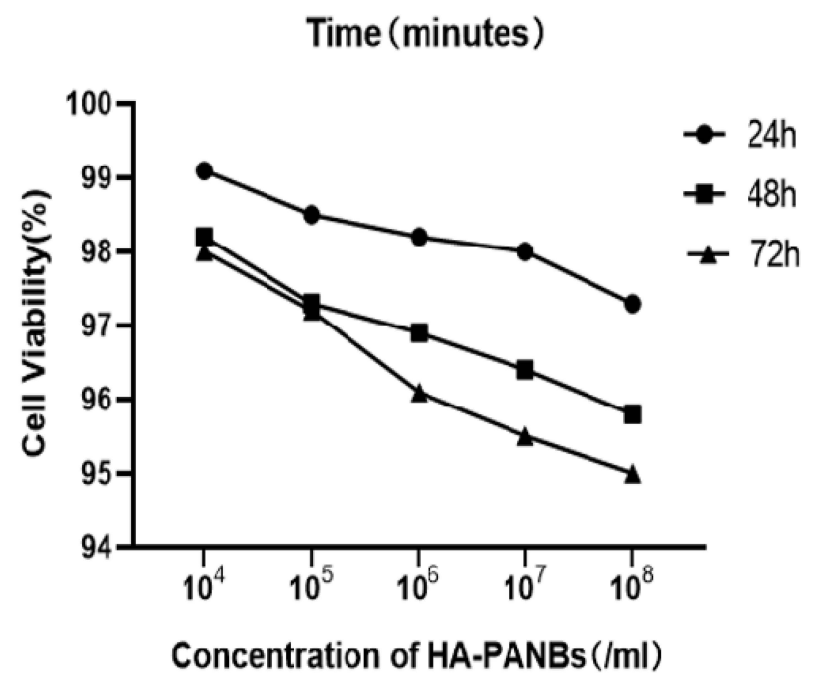

Figure 8 (A) HA-PANBs Changes of diameters with time; (B) HA-PANBs changes of concentration with time; (C) cytotoxicity changes with the concentration; (D) cytotoxicity changes over time. 


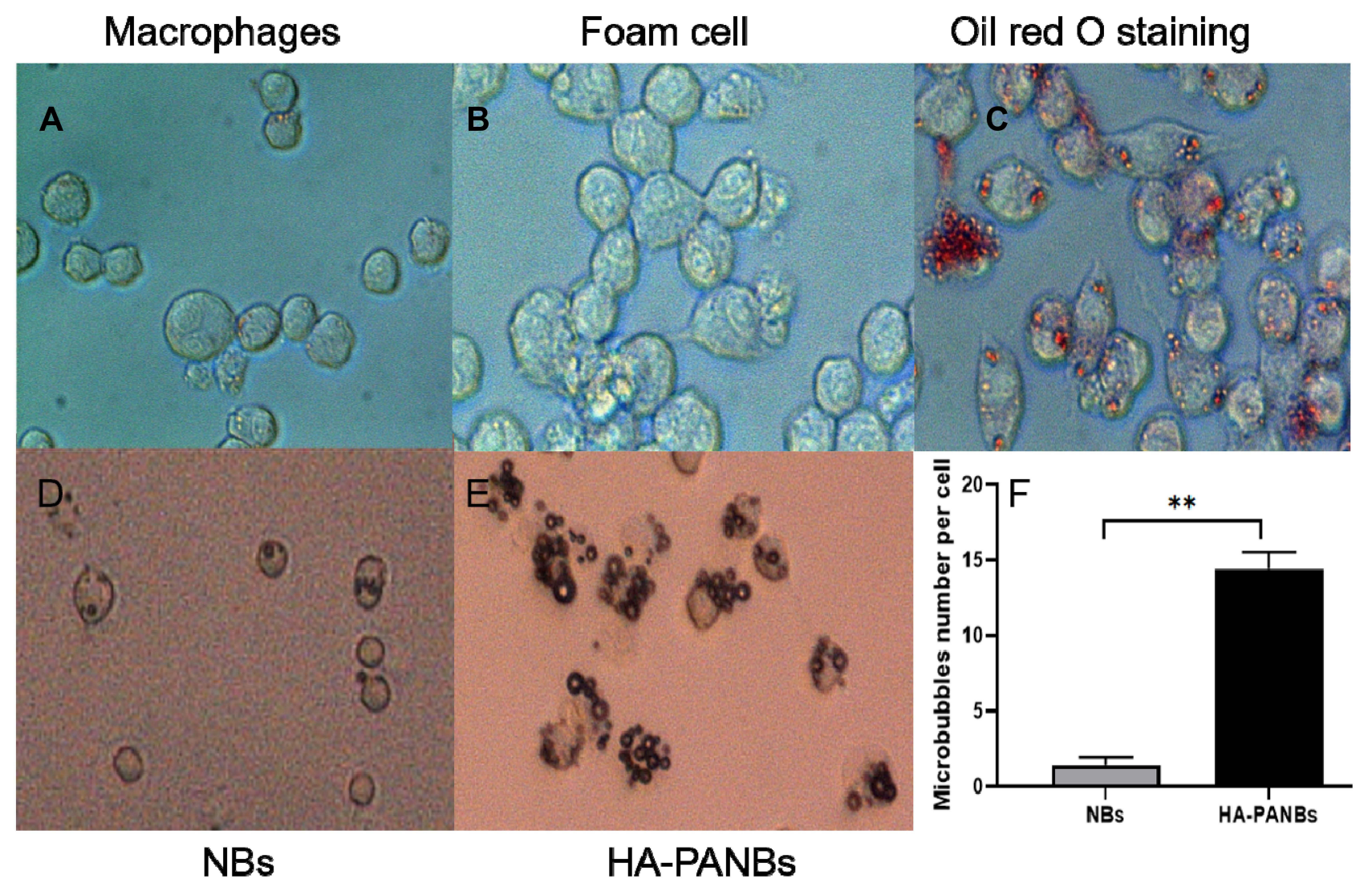

Figure 9 (A) Microscopic view of macrophages; (B) microscopic view of foam cell; (C) foam cell oil red O staining; (D) almost no NBs target to macrophages; (E) a large number of HA-PANBs targetto foam cells; $(\mathbf{F})$ quantitative results of cell targeting. $* * \mathrm{P}<0.00 \mathrm{I}$.
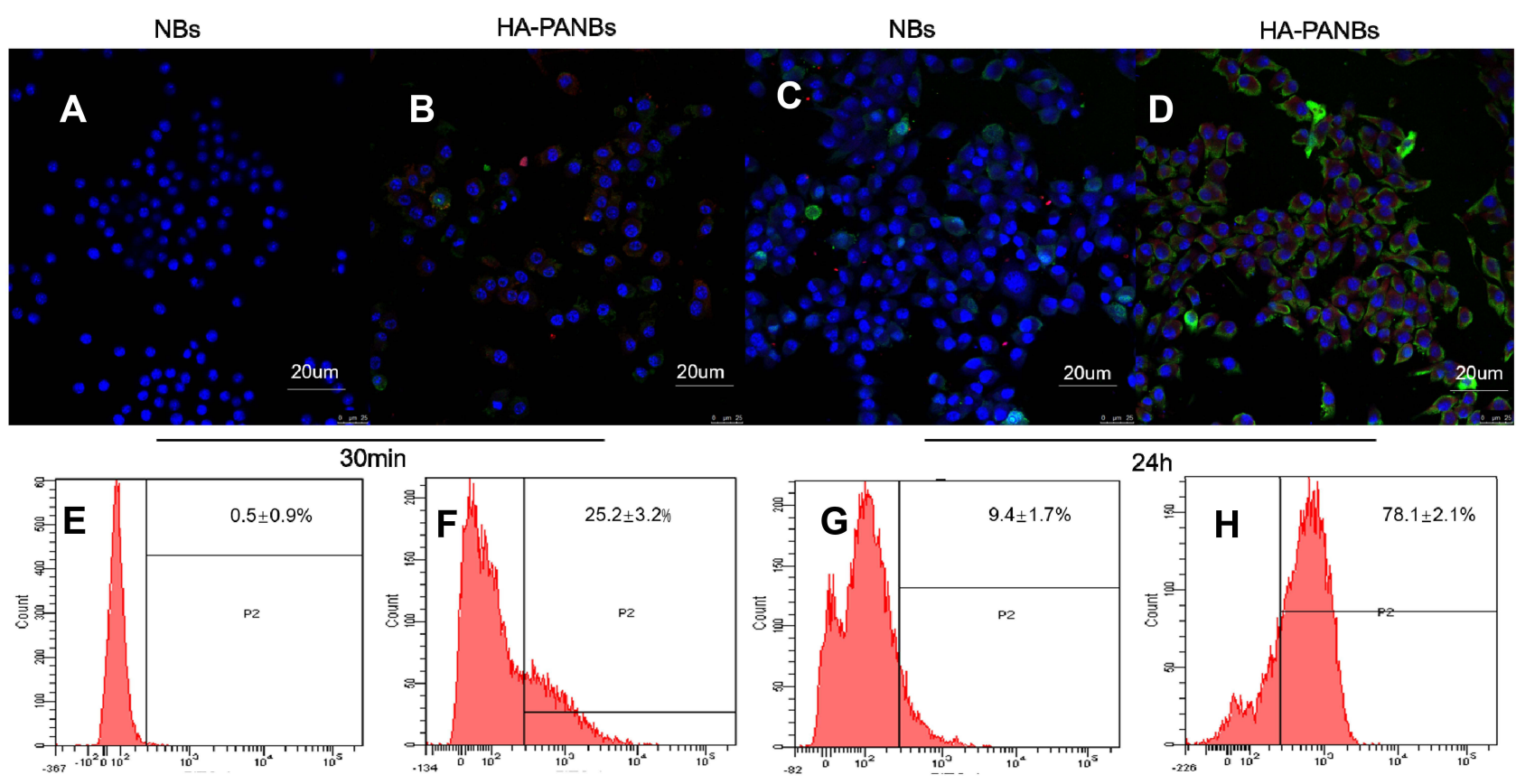

Figure 10 Laser confocal microscope observation of cell targeting results. (A) 30 minutes NBs group; (B) 30 minutes HA-PANBs group; (C) 24-hour NBs group; (D) 24hour HA-PANBs group.

Note: The following is the corresponding quantitative result. 


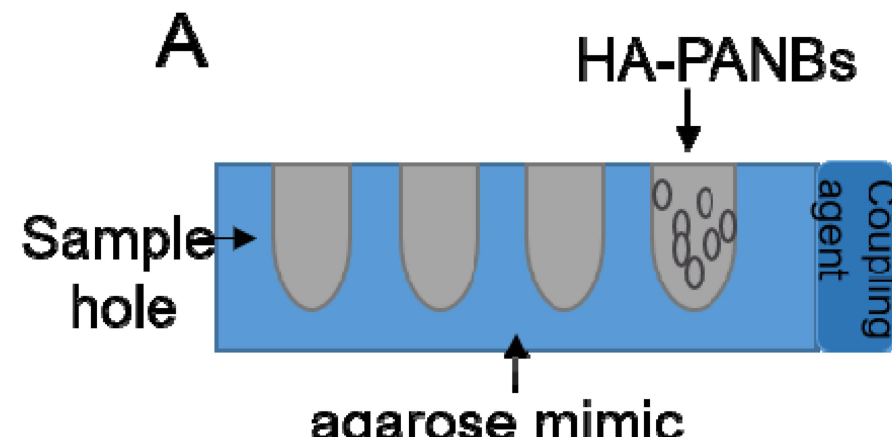

agarose mimic
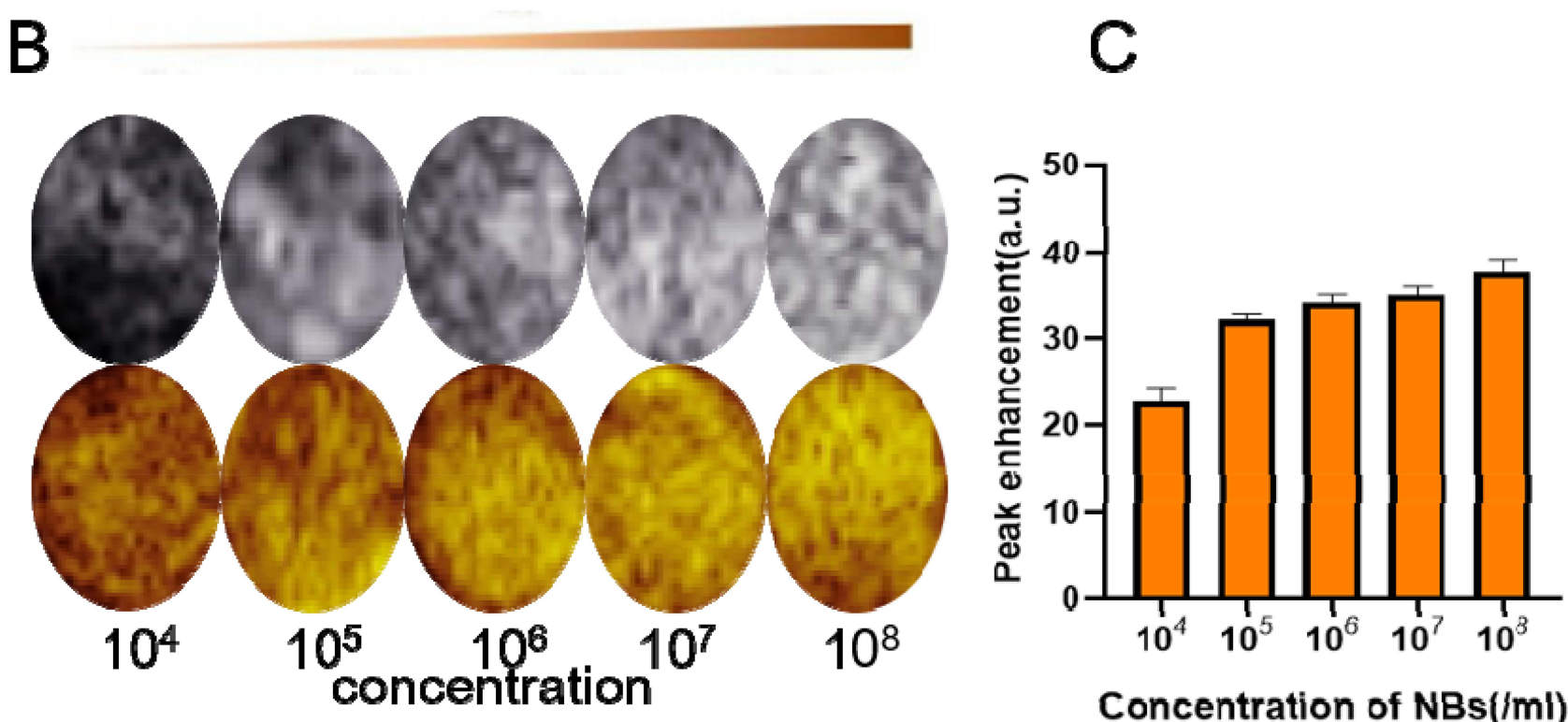

Concentration of $\mathrm{NBs}(/ \mathrm{ml})$

Figure I I Ultrasound images of HA-PANBs at different concentrations in vitro.

Notes: (A) Schematic diagram of the in vitro imaging method. (B) Two-dimensional and contrast-enhanced ultrasound images of HA-PANBs at different concentrations; (C) quantitative results.

advantages and disadvantages. In this experiment, when two targets and a therapeutic factor were carried at the same time, problems related to the molecular weight and competition between materials arose. ${ }^{19}$ Therefore, multiple connection methods applied in parallel, as well as a reliable method of ligand attachment, may be needed to produce microbubbles with ultrasound. In this experiment, the aptamers PM1 and ANM33 were attached to the surface of lipid microbubbles to construct a dual-target lipid microbubble carrier wrapped in HA as the outermost layer.

\section{Optimal Loading Capacity of PMI}

The most prominent advantage of targeted preparations is the maximization of the delivery of drugs to the target area, which can increase the local drug concentration and reduce the amount of drug used while avoiding side

Table 4 Contrast Signal from BNBs at Different Concentrations Over 60 Min

\begin{tabular}{|l|c|c|c|c|c|c|}
\hline concentration & $\mathbf{0 m i n}$ & $\mathbf{5 m i n}$ & $\mathbf{1 5} \mathbf{m i n}$ & $\mathbf{3 0 m i n}$ & $\mathbf{4 5 m i n}$ & $\mathbf{6 0 m i n}$ \\
\hline $10^{4}$ & $22.52 \pm 1.56$ & $22.41 \pm 1.25$ & $22.40 \pm 1.35$ & $22.29 \pm 1.28$ & $22.53 \pm 1.31$ & $22.45 \pm 1.38$ \\
$10^{5}$ & $34.38 \pm 1.20$ & $34.99 \pm 1.13$ & $34.59 \pm 1.13$ & $34.48 \pm 1.21$ & $34.92 \pm 0.88$ & $34.67 \pm 0.38$ \\
$10^{6}$ & $35.29 \pm 0.98$ & $35.25 \pm 0.86$ & $35.47 \pm 0.96$ & $34.88 \pm 0.91$ & $35.06 \pm 0.89$ & $35.39 \pm 0.24$ \\
$10^{7}$ & $34.89 \pm 0.93$ & $35.05 \pm 0.71$ & $35.27 \pm 0.84$ & $34.88 \pm 0.91$ & $35.06 \pm 0.89$ & $35.39 \pm 0.75$ \\
$10^{8}$ & $40.36 \pm 1.35$ & $40.38 \pm 1.07$ & $40.3 \pm 1.02$ & $40.28 \pm 1.02$ & $40.25 \pm 1.33$ & $40.15 \pm 1.45$ \\
\hline
\end{tabular}


effects. An aptamer is single-stranded DNA or RNA 20 to 200 bases in length that can specifically bind to a target substance through its spatial conformation and is currently recognized as the most promising target substance. ${ }^{17}$

The PM1 used in this experiment can specifically target macrophage-derived foam cells. ${ }^{20}$ The flow cytometry results showed that the extent of connection between PCNBs and PM1 increased as the amount of PM1 administered increased until reaching the maximum binding of $89.0 \pm 1.1 \%$, corresponding to $20 \mu \mathrm{L}$ of $10 \mu \mathrm{M}$ PM1 and a maximum binding amount of $2 \mu \mathrm{g} / 10^{8}$ microbubbles (Figure 4). In addition, the HS-SH-Oligo used in the construction of PM1 in this experiment was protected by disulfide bonds, so the modified PM1 still needed to be reduced before use; ${ }^{17}$ fortunately, the hydrolysis of maleimide is well known. However, qualitative and quantitative information on the stability and activity of the maleimide group during the preparation and in the final product is not available. The research results of Wang et $\mathrm{al}^{21}$ showed that hydrolysis of the maleimide group would hinder the functionalization of nanoparticles, and because the hydrolysate was maleic acid, the negative surface charge would be increased. In this experiment, a low-cytotoxicity cationic lipid membrane material (DC-Chol) was added; DC-Chol forms a phospholipid bilayer and imparts a positive charge to the surface. The cationic microbubble contrast agent CNB was successfully prepared, and after adding the first-level target PM1, the produced PCNBs maintained a positive potential. The nanoscale size, as shown in Table 2, enabled the connection of ANM33 in the subsequent step.

To further improve the efficiency of the connection to PM1, in this study, two common methods were used to prepare microbubbles: the preinsertion method, involving the insertion of DSPE-PEG2000-maleimide during the preparation of microbubbles, and the postinsertion method, involving the insertion of DSPE-PEG2000-maleimide after the preparation was completed. The maximum binding rate was $89.0 \pm 1.1 \%$ for the postinsertion method and $36.4 \pm 1.7 \%$ for the preinsertion method. The activity of the maleimide group was less affected by the postinsertion method than by the preinsertion method, as shown in Figure 5. The results of Oswald et $\mathrm{al}^{22}$ showed that $63 \%$ of active maleimide groups were retained on the surface of a product prepared using the preinsertion method, but the activity was significantly reduced to $32 \%$ in the subsequent purification. The postinsertion method had little effect on the activity of the maleimide group, and $76 \%$ of the maleimide group retained activity and could participate in the subsequent coupling reaction. The preparation method has a significant influence on the activity of the maleimide group. Similar results were obtained in this study, so subsequent experiments all used the postinsertion method.

\section{Optimal Carrying Capacity for ANM33}

Studies have found that mice can endure long-term ANM33 treatment but that miR-33 gene knockout mice develop systemic obesity and metabolic dysfunction, suggesting that ANM33 therapy has hidden risks. Because miR-33 plays a role in the development of $\mathrm{AS}$, it is involved in multiple biochemical processes, such as glucose metabolism, bile acid synthesis, cell proliferation, and endoplasmic reticulum stress. ${ }^{23}$ Therefore, the nonselective inhibition of systemic miR-33 with ANM33 is bound to cause serious systemic side effects, and accurate quantification is very important.

To quantify ANM33, the binding efficiency of ANM33 and PCNBs was detected at different concentrations with a microplate reader. The standard curve of ANM33 was good. After the optimal carrying efficiency of PM1 was determined, ANM33 was continuously injected according to the concentration gradient until 25 $\mu \mathrm{L}$ of $20 \mu \mathrm{M}$ ANM33 had been added. The UV absorbance of the PANBs no longer increased with increasing input, indicating that saturation had been reached. The maximum binding rate of PANBs was $62.7 \pm 2.1 \%$, and the maximum binding amount was $5 \mu \mathrm{g} / 10^{8}$ microbubbles (Table 6). Different amounts of PM1 were used to process the same amount of CNBs and react with the same amount of ANM33. The higher the proportion of PM1 was, the more ANM33 was incorporated into the PCNBs; the increase in ANM33 incorporation was rapid between PM1 proportions of 0.2 and 2 and slow between 2 and 3. As shown in Figure 7C, to reach a certain loading rate while also conserving materials, in the follow-up experiments, we used a PM1:ANM33 ratio of $1: 2$, that is, $2 \mu \mathrm{g}$ of PM1 and $4 \mu \mathrm{g}$ of ANM33.

To further clarify the connection between the material and NBs, confocal microscopy and flow cytometry were used to analyse the connection of PM1 and ANM33 with the CNBs. The surface of the cationic microbubbles under the laser confocal microscope emitted red fluorescence, indicating that Cy3-labelled ANM33 was successfully adsorbed. When the emission channel was switched, the surface of the microbubble in the field of view emitted green fluorescence, indicating that the AF488-labelled aptamer PM1 was successfully adsorbed. The synthesis channel was bright yellow (Figure 4), effectively eliminating the possibility of a false positive from the fluorescence microscope and thus confirming that PM1 and ANM33 
Table 5 Quantification of Different Concentrations of HAPANBs

\begin{tabular}{|l|c|}
\hline Concentration & Contrast Signal Intensity (a.u.) \\
\hline $10^{4}$ & $22.65 \pm 1.59$ \\
$10^{5}$ & $34.38 \pm 1.20^{*}$ \\
$10^{6}$ & $35.29 \pm 0.98^{* \#}$ \\
$10^{7}$ & $34.89 \pm 0.93^{* \#}$ \\
$10^{8}$ & $40.36 \pm 1.35^{* \# \alpha^{\wedge}}$ \\
$\mathrm{F}$ & 0.101 \\
$\mathrm{P}$ & $<0.001$ \\
\hline
\end{tabular}

Notes: *Compared with group $10^{4}, \mathrm{P}<0.05$; ${ }^{\#}$ compared with group $10^{5}, \mathrm{P}<0.05$; ${ }^{2}$ compared with group $10^{6}, \mathrm{P}<0.05$; $^{\wedge}$ compared with group $10^{7}, \mathrm{P}<0.05$.

Table 6 Quantification of the Binding Rates of PCNBs of Different Quality

\begin{tabular}{|l|c|}
\hline Group (ul) & Connection Rate (\%) \\
\hline 5 & $20.1 \pm 0.6$ \\
15 & $39.8 \pm 0.2^{*}$ \\
25 & $62.7 \pm 2.1^{* \#}$ \\
35 & $63.7 \pm 1.3^{* \#}$ \\
45 & $63.9 \pm 1.2^{* \#}$ \\
F & 31.77 \\
P & $<0.001$ \\
\hline
\end{tabular}

Notes: *Compared with group 5ul, $\mathrm{P}<0.05 ;{ }^{*}$ compared with group 15ul, $\mathrm{P}<0.05$.

were effectively coadsorbed on the surface of the cationic microbubbles. The extent of connection of ANM33 reached $62.7 \pm 2.1 \%$ after using electrostatic adsorption and covalent connections and $44.1 \pm 1.7 \%$ after using only electrostatic adsorption (Figure 6). Previous studies by Zhang ${ }^{24}$ and others showed that with electrostatic adsorption, $17.81 \pm 1.46 \mu \mathrm{g}$ of DNA can be carried per $5 \times 10^{8}$ microbubbles. In this study, although ANM33 also had a negative charge on its surface, it was still considerably different from traditional DNA. The connection rate was significantly lower with only electrostatic adsorption than with electrostatic adsorption combined with covalent connection. The results were significantly different for the two connection methods, and the PANBs maintained a positive potential after the addition of ANM33, as shown in Table 2. The above two materials had little effect on the NBs, and connection via sulfhydryl groups of maleimide, via electrostatic adsorption and via covalent bonds can realize the connection between the ultrasound microbubbles and the two ligands. The follow-up experiments are described next.

\section{Optimal HA Loading Capacity}

As the first-level target of this multistage hierarchical targeting, HA was coated on the surface of the PANBs through covalent linkages, which should effectively reduce the ineffective connections of preparations targeting the endothelial reticulum, connections to nontarget macrophages and intervention drug waste. ${ }^{12,19}$ Zhang et $\mathrm{al}^{11}$ used HA-coated targeting agents to effectively "escape" hepatic receptors by means of staged targeting and achieved efficient and precise targeting of macrophages in AS plaques.

At the end of this experiment, HA was coated on PANBs. The CTAB turbidimetric method can efficiently determine the HA content. ${ }^{11}$ The HA standard curve was good. After adding $20 \mu \mathrm{L}$ of $10 \mu \mathrm{M}$ PM1 and $25 \mu \mathrm{L}$ of 20 $\mu \mathrm{M}$ ANM33 to obtain PANBs, $3 \mu \mathrm{g}, 5 \mu \mathrm{g}, 7 \mu \mathrm{g}, 9 \mu \mathrm{g}$, or $11 \mu \mathrm{g}$ HA was added to obtain the final HA-PANBs. The absorbance at $550 \mathrm{~nm}$ by the microplate reader reached saturation at $7 \mu \mathrm{g}$ of HA, at which point it stopped increasing, as shown in Figure 7E.

We carried out related experiments to assess the effective connection between the carboxyl group on HA and the amino group on PANBs. The connection to HA is the last step of the preparation. Due to the stability of the chemical bonds that it can form, the carboxylic acid residue of HA can also serve as a functional group for covalent bonding: the proportion of connected HA was $61.4 \pm 2.18 \%$ when EDC and NHS were used for activation, as shown in Figure 7B, but only 26.9 $\pm 2.23 \%$ when only electrostatic adsorption was used, reflecting a nearly 3 -fold difference. In addition to the CTAB turbidimetric method for determining the effective connection of covalent bonds, a change in particle size from the nanometre to the micrometre scale also confirmed the effective connection of HA. ${ }^{25}$ Although their particle size increased, the microbubbles remained smaller than red blood cells. HA affected the potential of the microbubbles, which is important for ensuring passage through the capillary network and engulfment by macrophages. After HA was connected, the potential of the microbubble surface changed from positive to negative. However, when the cationic lipid membrane material DC-Chol was not added, the potential changed from $-23.07 \pm 2.18$ to $-5.51 \pm 1.87$ upon HA connection, and this difference was statistically significant. The effective construction of a cationic carrier core is important for subsequent experiments and results.

In view of the advantages and disadvantages of singletarget ultrasound microbubbles and single-connection methods to construct ultrasound microbubbles, ${ }^{26}$ DC-Chol, a cationic lipid membrane material with low cytotoxicity, ${ }^{24}$ was added to form a bilayer of phospholipids while maintaining a positive surface charge, and cationic microbubble 
contrast agent CNBs were successfully prepared. The postinsertion connection of PM1 retained the stability and activity of the maleimide group to a large extent, and $89.0 \pm 1.1 \%$ of the added PM1 was connected, which provides an experimental foundation for the effective connection of other aptamers. The extent of the ANM33 connection after electrostatic adsorption combined with covalent connections was 10 times higher than that for ordinary NBs. Thus, this combined method overcomes the low gene-carrying capacity and low transfection efficiency of microbubbles ${ }^{11,27}$ that result from pure electrostatic adsorption. Thus, the developed method significantly improves the connection extent. HA-PANBs were formed by coupling HA and PANBs via covalent connections. The HA-PANB dual-target microbubble contrast agent was formed by coating PANBs with HA. The microbubbles in each test group were uniform in size and had good dispersion and stability.

In this study, a simple in vitro foam cell model was prepared with reference to the study of Zhang. ${ }^{11}$ Oil red $\mathrm{O}$ staining results showed lipid droplet formation when the ox-ldl intervention amount reached $60 \mathrm{mg} / \mathrm{L}$. In terms of cell targeting, in this study, NBs and HA-PANBs were coincubated with foam cell models and verified by ordinary optical microscopy, laser confocal microscopy and flow cytometry.

The results showed that under the inverted microscope, a large number of microbubbles (MBs) were clustered around the cells in the HA-PANB group, while few MBs could be seen in the space between cells, which indicated that the targeted MBs had a high affinity for foam cells; additionally, there were no or only a few MBs around the NB group. Quantitative analysis showed that the number of MBs gathered around foam cells in the HA-PANBS group was 7.2 times that in the NBs group. Under a fluorescence microscope, more red and green fluorescence expression was also observed in the HA-PANB group. Flow cytometry showed that the binding rate of HA-PANBs to damaged macrophages was significantly higher than that of nontargeted nanovesicles. The results of this study preliminarily demonstrated that HA-PANBs had obvious targeting and high affinity for damaged macrophages in the process of AS.

As gene vectors, the biological safety of the materials must be guaranteed. Therefore, the CCK- 8 method was first used to evaluate the cytotoxicity of HA-PANBs in vitro in part of this study. The composition of NBs used in this study was similar to that of SonoVue, an ultrasound contrast agent that has been routinely used in clinical practice, and it has been shown that HA-PANBs do not cause any damage to cells at normal doses. Therefore, the above results fully demonstrate the safety of HA-PANBs in vitro.

The contrast was as expected. With increasing concentration, the signal of the HA-PANB solution was gradually enhanced. However, it is worth emphasizing that we also found that although the contrast signal in the near field of the ultrasound beam does increase with increasing concentration, especially when the concentration reaches $10^{8}$, the signal attenuation in the region behind the strong echo signal is very obvious. Through further comparison, we found that when the ultrasonic probe frequency was 18 $\mathrm{MHz}$ and the concentration was $10^{6} \mathrm{BNBs}$, uniform ceUS signals could be seen in the simulated sample addition hole, and the in vitro contrast effect was the most ideal among the five concentrations.

In summary, this experiment determined the maximum loading capacity of each therapeutic factor through step-bystep analysis of the inside to the outside of the complex. We believe that angiography can be achieved using microbubbles with a 2:4:7 PM1:ANM33:HA loading, which have a high capacity for ANM33 and stable physical properties and can significantly enhance the imaging and targeting of damaged macrophages in vitro, thereby laying the foundation for further high-efficiency transfection of antisense oligonucleotides in vivo and in vitro. Both targets and therapeutic factors can be effectively connected to the NB core of the carrier, making it an ideal ultrasound contrast agent.

\section{Conclusion}

A variety of connection methods were used to successfully connect ultrasound microbubbles to the aptamers PM1, ANM33 and HA to effectively produce HA-PANBs. The developed method combines the advantages and disadvantages of previous connection methods and single targets and can be used for AS targets. Gene therapy based on this method represents a new vector and transfection method.

\section{Funding}

The authors gratefully acknowledge the support of this research by the National Natural Science Foundation of China (Grant No.82060321), the National Natural Science Foundation of China (Grant No.32071459), "State Key Laboratory of Pathogenesis, Prevention and Treatment of High Incidence Diseases in Central Asia Fund" (Grant No. SKL-HIDCA-2021-XXG6), Xinjiang Medical University Postgraduate Scientific Research innovation Project (Grant No.XJ2021G200). 


\section{Disclosure}

The authors report no conflicts of interest in this work.

\section{References}

1. Tajbakhsh A, Rezaee M, Kovanen PT, et al. Efferocytosis in atherosclerotic lesions: malfunctioning regulatory pathways and control mechanisms. Pharmacol Ther. 2018;188:12-25. doi:10.1016/j. pharmthera.2018.02.003

2. Huang L, Chambliss KL, Gao X, et al. SR-B1 drives endothelial cell LDL transcytosis via DOCK4 to promote atherosclerosis. Nature. 2019;569(7757):565-569. doi:10.1038/s41586-019-1140-4

3. Back M, Yurdagul A, Tabas I, et al. Inflammation and its resolution in atherosclerosis: mediators and therapeutic opportunities. Nat Rev Cardiol. 2019;16(7):389-406.

4. Price NL, Fernandez-Hernando C. Novel role of miR-33 in regulating of mitochondrial function. Circ Res. 2015;117(3):225-228. doi:10.1161/CIRCRESAHA.117.306949

5. Ouimet M, Ediriweera H, Afonso MS, et al. microRNA-33 regulates macrophage autophagy in atherosclerosis. Arterioscler Thromb Vasc Biol. 2017;37(6):1058-1067. doi:10.1161/ATVBAHA.116.308916

6. Price NL, Rotllan N, Zhang X, et al. Specific disruption of Abcal targeting largely mimics the effects of miR-33 knockout on macrophage cholesterol efflux and atherosclerotic plaque development. Circ Res. 2019;124(6):874-880. doi:10.1161/CIRCRESAHA.118.314415

7. Rahman MS, Woollard K. Atherosclerosis. Adv Exp Med Biol. 2017;1003:121-144.

8. Ahmed M, Gustafsson B, Aldi S, et al. Molecular imaging of a new multimodal microbubble for adhesion molecule targeting. Cell Mol Bioeng. 2018;12(1):15-32. doi:10.1007/s12195-018-00562-z

9. Fei Y, Yu S, Yang M, et al. Ultrasound molecular imaging of atherosclerosis for early diagnosis and therapeutic evaluation through leucocyte-like multiple targeted microbubbles. Theranostics. 2018;8 (7):1879-1891. doi:10.7150/thno.22070

10. Cordray MS, Amdahl M, Richards-Kortum RR. Gold nanoparticle aggregation for quantification of oligonucleotides: optimization and increased dynamic range. Anal Biochem. 2012;431(2):99-105. doi:10.1016/j.ab.2012.09.013

11. Zhang M, He J, Jiang C, et al. Plaque-hyaluronidase-responsive high-density-lipoprotein-mimetic nanoparticles for multistage intimal-macrophage-targeted drug delivery and enhanced anti-atherosclerotic therapy. Int $J$ Nanomed. 2017;12:533-558. doi:10.2147/IJN.S124252

12. Punjabi M, Xu L, Ochoa-Espinosa A, et al. Ultrasound molecular imaging of atherosclerosis with nanobodies: translatable microbubble targeting murine and human VCAM (Vascular Cell Adhesion Molecule) 1. Arterioscler Thromb Vasc Biol. 2019;39(12):2520-2530. doi:10.1161/ATVBAHA.119.313088

13. Shentu WH, Yan CX, Liu CM, et al. Use of cationic microbubbles targeted to P-selectin to improve ultrasound-mediated gene transfection of hVEGF165 to the ischemic myocardium. J Zhejiang Univ Sci B. 2018;19(9):699-707. doi:10.1631/jzus.B1700298

International Journal of Nanomedicine

\section{Publish your work in this journal}

The International Journal of Nanomedicine is an international, peerreviewed journal focusing on the application of nanotechnology in diagnostics, therapeutics, and drug delivery systems throughout the biomedical field. This journal is indexed on PubMed Central, MedLine, CAS, SciSearch ${ }^{\mathbb{}}$, Current Contents ${ }^{\mathbb{B}} /$ Clinical Medicine, $^{2}$
14. Libby P, Loscalzo J, Ridker PM, et al. Inflammation, immunity, and infection in atherothrombosis: JACC review topic of the week. $J \mathrm{Am}$ Coll Cardiol. 2018;72(17):2071-2081. doi:10.1016/j.jacc.2018. 08.1043

15. Moore KJ, Sheedy FJ, Fisher EA, et al. Fisher Macrophages in atherosclerosis: a dynamic balance. Nat Rev Immunol. 2013;13 (10):709-721. doi:10.1038/nri3520

16. Wang S, Hossack JA, Klibanov AL. Targeting of microbubbles: contrast agents for ultrasound molecular imaging. J Drug Target. 2018;26(5-6):420-434. doi:10.1080/1061186X.2017.1419362

17. Yang X, Zhao J, Duan S, et al. Enhanced cytotoxic T lymphocytes recruitment targeting tumor vasculatures by endoglin aptamer and IP-10 plasmid presenting liposome-based nanocarriers. Theranostics. 2019;9(14):4066-4083. doi:10.7150/thno.33383

18. Bose RJC, Uday Kumar S, Zeng Y, et al. Tumor cell-derived extracellular vesicle-coated nanocarriers: an efficient theranostic platform for the cancer-specific delivery of anti-miR-21 and imaging agents. ACS Nano. 2018;12(11):10817-10832. doi:10.1021/acsnano.8b02587

19. Shamloo A, Ebrahimi S, Amani A, et al. Targeted drug delivery of microbubble to arrest abdominal aortic aneurysm development: a simulation study towards optimized microbubble design. Sci Rep. 2020;10(1):5393. doi:10.1038/s41598-020-62410-3

20. Yan PK, Wang JB, Zhang H, et al. Research on the specificity of PMl aptamer about THP-1 macrophage derived foam cells. Chin J Arterioscleros. 2011;19(5):405-408.

21. Wang CH, Huang YF, Yeh CK. Aptamer-conjugated nanobubbles for targeted ultrasound molecular imaging. Langmuir. 2011;27 (11):6971-6976. doi:10.1021/la2011259

22. Oswald M, Geissler S, Goepferich A. Determination of the activity of maleimide-functionalized phospholipids during preparation of liposomes. Int $J$ Pharm. 2016;514(1):93-102. doi:10.1016/j. ijpharm.2016.06.116

23. Price NL, Singh AK, Rotllan N, et al. Genetic ablation of miR-33 increases food intake, enhances adipose tissue expansion, and promotes obesity and insulin resistance. Cell Rep. 2018;22 (8):2133-2145. doi:10.1016/j.celrep.2018.01.074

24. Zhang L, Sun Z, Ren P, et al. Localized delivery of shRNA against PHD2 protects the heart from acute myocardial infarction through ultrasound-targeted cationic microbubble destruction. Theranostics. 2017;7(1):51-66. doi:10.7150/thno.16074

25. Xie S, Li G, Hou Y, et al. A synergistic bactericidal effect of low-frequency and low-intensity ultrasound combined with levofloxacin-loaded PLGA nanoparticles on $M$. smegmatis in macrophages. J Nanobiotechnology. 2020;18(1):107. doi:10.1186/ s12951-020-00658-7

26. Liu L, He H, Zhang M, Zhang S, Zhang W, Liu J. Hyaluronic acid-decorated reconstituted high density lipoprotein targeting atherosclerotic lesions. Biomaterials. 2014;35(27):8002-8014. doi:10.1016/j. biomaterials.2014.05.081

27. Liu Y, Zhou Y, Xu J, et al. Ultrasound molecular imaging-guided tumor gene therapy through dual-targeted cationic microbubbles. Biomater Sci. 2021;9(7):2454-2466. doi:10.1039/D0BM01857K

Journal Citation Reports/Science Edition, EMBase, Scopus and the Elsevier Bibliographic databases. The manuscript management system is completely online and includes a very quick and fair peer-review system, which is all easy to use. Visit http://www.dovepress.com/ testimonials.php to read real quotes from published authors. 